\title{
H. pylori Eradication Treatment Causes Alterations in the Gut Microbiota and Blood Lipid Levels
}

\author{
Gracia M. Martín-Núñez ${ }^{1,2 \star \dagger}$, Isabel Cornejo-Pareja ${ }^{1,2+}$, M. del Mar Roca-Rodríguez ${ }^{3}$, \\ Mercedes Clemente-Postigo ${ }^{2,4}$, Fernando Cardona ${ }^{1,2}$, José C. Fernández-García ${ }^{1,2}$, \\ Isabel Moreno-Indias ${ }^{1,2 \star}$ and Francisco J. Tinahones ${ }^{1,2}$ \\ ${ }^{1}$ Unidad de Endocrinología y Nutrición, Hospital Universitario Virgen de la Victoria, Málaga, Spain, ${ }^{2}$ Centro de Investigación \\ Biomédica en Red de Fisiopatología de la Obesidad y la Nutrición (CIBEROBN), Instituto de Salud Carlos III, Madrid, Spain, \\ ${ }^{3}$ Departamento de Endocrinología y Nutrición, Hospital Universitario Puerta del Mar, Cádiz, Spain, ${ }^{4}$ Departamento de \\ Biología Celular, Fisiología e Inmunología, Instituto Maimónides de Investigación Biomédica de Córdoba (IMIBIC), \\ Universidad de Córdoba, Hospital Universitario Reina Sofía, Córdoba, Spain
}

\section{OPEN ACCESS}

Edited by:

Giovanni Tarantino,

University of Naples Federico II, Italy

Reviewed by:

Mitsushige Sugimoto,

Shiga University of Medical

Science, Japan

Timothy Powell,

University of Oxford, United Kingdom

*Correspondence:

Gracia M. Martín-Núñez graciamaria_mn@hotmail.com

Isabel Moreno-Indias

isabel_moreno_indias@hotmail.com

${ }^{\dagger}$ These authors have contributed equally to this work

Specialty section:

This article was submitted to

Translational Medicine,

a section of the journal

Frontiers in Medicine

Received: 16 March 2020

Accepted: 30 June 2020

Published: 11 August 2020

Citation:

Martín-Núñez GM, Cornejo-Pareja I,

Roca-Rodríguez MM Clemente-Postigo M, Cardona F

Fernández-García JC, Moreno-Indias I and Tinahones FJ (2020) H. pylori

Eradication Treatment Causes Alterations in the Gut Microbiota and Blood Lipid Levels. Front. Med. 7:417.

doi: 10.3389/fmed.2020.00417
Background: The gut microbiome plays an important role in the lipid metabolism. Antibiotic treatment causes changes in the intestinal microbiota. Our objective was to explore the relationship between changes in the intestinal microbiota and the level of plasma high density lipoprotein cholesterol (HDL) and low density lipoprotein cholesterol (LDL).

Methods: Prospective case-control study with Helicobacter pylori-positive patients undergoing eradication therapy with omeprazole, clarithromycin, and amoxicillin. Stool and blood samples were obtained from 20 controls $(H$. pylori negative) and 40 patients before and 2 months after antibiotic treatment. Gut microbiota was determined through $16 \mathrm{~S}$ rRNA amplicon sequencing (Illumina MiSeq).

Results: Eradication treatment for $H$. pylori increased the HDL levels, and caused changes in gut microbiota profiles. An unfavorable lipid profiles (high LDL and low HDL levels) was associated with a low microbial richness and an increase of the Bacteroidetes phylum. Prevotella copri, Lachonobacterium, and Delsufovibrio were positively associated with HDL while Rikenellaceae was negatively associated with HDL after completing antibiotic treatment.

Conclusions: Helicobacter pylori eradication treatment could improve lipid metabolism in relation with an increase in the HDL. Changes in the abundance of specific bacteria, such as P. copri, Lachonobacterium, Delsufovibrio, and Rikenellaceae could be associated with change in the plasma HDL levels.

Keywords: LDL, HDL, gut microbiota, antibiotic, H. pylori

\section{INTRODUCTION}

Increasing evidence has demonstrated that the intestinal microbiota is critical for the development of diseases associated with altered lipid metabolism (1) including variation in the level of blood lipids (2). The microbiome affects ability to metabolize lipids by the host taking part in the absorption, storage, and energy resulted from the diet (3), because of its ability to convert 
bile acids and produce short-chain fatty acids (SCFAs) within the intestinal lumen $(4,5)$.

Triglycerides and high density lipoproteins cholesterol (HDL) levels have been associated to the diversity and amount of Proteobacteria and Bacteroidetes members (including Christensenellaceae, Pasteurellaceae, and genus Butyricimonas) (2). HDL function is to transport cholesterol to the liver from peripheral tissues for its subsequent excretion as biliary sales, and to endocrine organs for the synthesis of steroid hormones. The concentration of serum low-density lipoproteins cholesterol (LDL) is regulated, in part, by a cross-talk between the absorption of dietary and biliary cholesterol in the intestine, and the biosynthesis of cholesterol in the liver (6). LDL and HDL levels are biomarkers associated with the CVD risk (7).

Helicobacter pylori is a Gram-negative bacterium that colonizes the gastric mucosa commonly causing a chronic infection. Also, it has been associated with extradigestive pathologies including coronary heart disease (8). H. pylori eradication through oral administration of antibiotic and a proton-pump inhibitor has been associated with alterations in the gut microbiota $(7,9)$. We have previously found that antibiotic therapy used for $H$. pylori eradication can alter the intestinal microbiota population, and more importantly, these changes were associated to glucose metabolism and GLP-1 $(10,11)$. However, there is scarce data in humans about the relationship between blood lipids and changes in the profile of gut microbiota after antibiotic therapy (12). Thus, in the present study, our objective was to test the relationship between changes in the gut microbiota and blood lipids level in patients who received antibiotic treatment for $H$. pylori eradication.

\section{MATERIALS AND METHODS}

\section{Study Subjects and Design}

Forty volunteers with positive $H$. pylori antigen in faeces tested by immunochromatography were derived from the Microbiology Unit of the Virgen de la Victoria Hospital (Málaga, Spain). Sample size was calculated taking into account a reduction in richness (according to Chaol index) of 16\% after the antibiotic therapy $(13,14)$. The inclusion criteria were: (1) adulthood (1865 years old), and (2) primary infection with $H$. pylori. Besides, a group of non-infected volunteers (20 individuals) was included as control. Exclusion criteria were (1) diabetes (type 1 or 2); (2) prior H. pylori documented treatment; (3) antibiotherapy 3 months before recruitment; and (4) without informed consent.

The study included two visits, before and 60 days posttreatment (20 mg omeprazole, $500 \mathrm{mg}$ clarithromycin, and $1000 \mathrm{mg}$ amoxicillin, twice daily during 10 days) for patients, while a unique visit was assessed in the case of the control volunteers. Visitations comprised a physical exploration, a fasting blood sample, and an oral glucose tolerance test (OGTT) with $75 \mathrm{~g}$ glucose and measurements at 30,60, and $120 \mathrm{~min}$. Moreover, samples of stool were obtained in every visit and immediately frozen at $-80^{\circ} \mathrm{C}$. The investigation protocol was conducted

Abbreviations: SCFAs, short-chain fatty acids; HDL, high density lipoproteins; LDL, low-density lipoproteins; OGTT, glucose tolerance test. consonantly with the Declaration of Helsinki and conveniently approved by the Medical Ethics Committee at Virgen de la Victoria University Hospital. Volunteers were supplied with a written informed consent, as well as they were advised of the study characteristics.

\section{Anthropometric, Biochemical, and Dietetics Measurements}

Anthropometric measurements (height, weight, and waist and hip circumferences) were recorded (15). An enzymatic method was used for the measurement of triglycerides $(\mathrm{mmol} / \mathrm{L})$, total cholesterol (mg/dl), and HDL (mg/dl) (Randox Laboratories Ltd.), while C-reactive protein (CRP) was measured with a Dimension autoanalyzer (Dade Behring Inc.). LDL (mg/dl) was determined through the Friedewald formula. Food intake was evaluated with 7/24-h dietary recalls. The dietary variables (fats, fiber, proteins, etc.) were determined using DIAL $\left.^{(}\right)$nutrition software and the Diet Balancer software (Cardinal Health Systems Inc.).

\section{Gut Microbiota Analysis}

The determination of the gut microbiota was assessed as was described previously (11). In brief, the QIAamp DNA Stool Mini Kit was used for the extraction of DNA from fecal samples, and posteriorly the DNA concentrations were determined by Qubit ${ }^{\circledR}$ Fluorometric (Thermo Fisher Scientific). The 16S rRNA V3-V4 amplicon was analyzed with the universal primers reported by Klindworth et al. (16). The amplicon size $\sim 460$ bp was verified with a bioanalyzer (Agilent 2100, USA). AMPure XP beads (Beckman Coulter Genomic, CA, USA) were used to purify amplicon products. Samples were multiplexed with Nextera XT Index Kit (Illumina, CA, USA). Illumina MiSeq platform (Illumina, San Diego, USA) was used for the paired-end sequencing of amplicons.

Quantitative Insights into Microbial Ecology (Qiime) tool (version 1.9.1, open source software) was used for the analysis of the merged paired-end reads. The operational taxonomic units (OTUs) were generated at $97 \%$ similarity and alignment by UCLUST consensus using the Greengenes 16S rRNA gene database. Alpha diversity (Chaol and Shannon indixes, as well as the observed species) was also assessed with Qiime. Alpha diversity was evaluated with the rarefaction workflow, with a threshold of 34,385 sequences. Rarefied data were used for the downstream analysis. OTUs in less than five different samples were excluded. Raw data is sored at the public repository SRA database (NCBI) with the BioProject PRJNA517270.

\section{Statistical Analysis}

SPSS 22.0 (SPSS Inc., Chicago, IL, USA) and QIIME (version 1.9.1; open source software) were used for the statistical analysis. The data were expressed as mean \pm standard deviation. Statistical comparisons between the means for independent samples and paired samples (before and after antibiotic eradication treatment) was performed using the Student's $t$-test. Mann-Whitney and Wilcoxon signed-rank tests were used for the evaluation of nonparametric variables. Correlation analysis between analytical, clinical, and microbial populations variables was analyzed using 
the Spearman bivariate correlations test. Linear regression models were applied to identify bacterial changes as independent predictors of the selected variables (HDL/LDL ratio and HDL-). A $p<0.05$ treshold was established for the statistical significance.

\section{RESULTS}

\section{Lipid Metabolism}

The anthropometric and clinical variables of the patients and control subjects have been previously described (11). Anthropometric variables as BMI and waist circumference or biochemical parameters as triglycerides and cholesterol level did not reach statistical differences between groups. Focusing on lipid metabolism, HDL levels increased significantly after $H$. pylori eradication with antibiotic therapy $(p=0.021)$, while LDL levels were significantly lower in controls than in $H$. pylori-infected subjects $(p=0.036$; Table 1$)$. In addition, HDL/LDL ratio was significantly lower in patients pre- and post- $H$. pylori eradication than in control subjects $(p=0.007$ and $p=0.03$, respectively; Table 1). HDL/LDL ratio and HDL were associated with BMI in a regression model $\left(R^{2}=0.079, \beta=-0.281, p=0.005 ; R^{2}=0.93\right.$, $\beta=-0.305, p=0.003$, respectively). No statistically differences in the dietary intake energy, micro or macronutrients and dietary fiber between patients and controls $(p>0.05)$ (data not shown).

\section{Gut Microbiome and Lipids Metabolism}

Our H. pylori-positive patients study model who received antibiotics for a limited period of time has allowed us to evaluate changes in the microbiota, HDL and LDL, avoiding the confounding factors derived from the presence of other diseases, or metabolic alterations.

TABLE 1 | Characteristics of the study groups.

\begin{tabular}{lccc}
\hline Variables & $\begin{array}{c}\text { Pre-H. pylori } \\
\text { eradication } \\
(\boldsymbol{n}=\mathbf{4 0})\end{array}$ & $\begin{array}{c}\text { Post- } \boldsymbol{H} \text {. pylori } \\
\text { eradication } \\
(\boldsymbol{n}=\mathbf{4 0})\end{array}$ & $\begin{array}{c}\text { Control group } \\
(\boldsymbol{n}=\mathbf{2 0})\end{array}$ \\
\hline Age (years) & $46.95 \pm 12.78$ & $46.95 \pm 12.78$ & $43.86 \pm 12.63$ \\
Men/women (n) & $16 / 24$ & $16 / 24$ & $9 / 13$ \\
BMl (kg/m²) & $26.92 \pm 4.30$ & $26.91 \pm 4.40$ & $25.89 \pm 4.54$ \\
Waist (cm) & $92.10 \pm 12.06$ & $91.27 \pm 11.73$ & $89.8 \pm 13.23$ \\
HDL (mg/dL) & $52.97 \pm 12.9^{\mathrm{a}}$ & $55.36 \pm 16.36^{\mathrm{a}}$ & $57 \pm 15.8$ \\
LDL (mg/dL) & $121.45 \pm 35.8^{\mathrm{a}}$ & $117.96 \pm 33.4$ & $102.05 \pm 34^{\mathrm{a}}$ \\
HDL/LDL & $0.47 \pm 0.17^{\mathrm{b}}$ & $0.51 \pm 0.22^{\mathrm{a}}$ & $0.62 \pm 0.25^{\mathrm{b}, \mathrm{a}}$ \\
Triglycerides (mg/dL) & $97.2 \pm 39.6$ & $93.5 \pm 36.4$ & $89.70 \pm 41.78$ \\
Cholesterol (mg/dL) & $194.22 \pm 40.84$ & $191.34 \pm 37.15$ & $177.05 \pm 39.5$ \\
DBP (mmHg) & $77.75 \pm 9.58$ & $80.50 \pm 11.37$ & $75.95 \pm 10$ \\
SBP (mmHg) & $123.84 \pm 16.62$ & $125.42 \pm 21.36$ & $120.3 \pm 13.35$ \\
CRP (mg/L) & $4.07 \pm 2.44$ & $3.56 \pm 2.11$ & $4.14 \pm 2.92$ \\
\hline
\end{tabular}

All values are means \pm standard deviations. Wilcoxon's test was used to compare patients before and after $\mathrm{H}$. pylori eradication. The Mann-Whitney U-test or Student's t-test were used to compare independent group. Equal letter indicates statistical signiticative differences in the mean between those 2 groups, $p<0.05$. BMI, body mass index; HDL, high-density lipoprotein; LDL, low-density lipoprotein; TGs, triglycerides, TC, total cholesterol; DBP, diastolic blood pressure; SBP, systolic blood pressure; CRP, C-reactive protein.
Helicobacter pylori infection and its eradication with antibiotic affected alpha diversity (Chao 1 and Shannon indexes) and this data has been previously described by our group (11). Control subjects showed a greatest diversity and richness, with statistically significant differences, compared to the $H$. pylori patients (pre- and post-eradication treatment) $(p<0.05)(11)$. Chaol index correlated positively with the HDL/LDL ratio $(r=$ $0.258, p=0.04)$.

According to the abundance of each identified OTU, the most abundant bacterial phyla were Firmicutes and Bacteroidetes, while Actinobacteria, Proteobacteria, and Verrucomicrobia contributed with smaller proportions (1-5\%). Changes in the microbiome between these subjects has been described in detail by our group in a previous work (11). The phylum Bacteroidetes, that was greater in patients $(58.72 \pm 13.62$ and $63.50 \pm 10.30 \mathrm{vs}$. $45.89 \pm 13.57)$, showed negative correlations with the HDL/LDL ratio ( $r=-0.237, p=0.021$ ), while the phylum Firmicutes, which was greater in controls $(45.68 \pm 15.61$ vs. $35.29 \pm 11.82$ and $32.06 \pm 10.29, p<0.05)$, showed positive correlations with the HDL/LDL ratio $(r=0.230, p=0.025)$ in the studied groups. In the regression analysis, Bacteroidetes and Firmicutes predicted changes in the HDL/LDL ratio (Figure 1).

Genera and species of the phyla Bacteroidetes and Firmicutes showed association with the HDL/LDL ratio (Table 2). Bacteroides coprophilus, Eubacterium, and E. biforme predicted changes in the HDL/LDL ratio, in a linear regression model adjusted for age, sex, and BMI (Table 2). The presence of these bacteria was higher in subjects with $H$. pylori infection compared to controls ( $p<0.05$, data not shown). The associations shown in the Table 2 were found in patients and controls.

We evaluated the impact of antibiotic eradication therapy on the abundance of identified OTU, LDL, and HDL level. Significant correlations between changes in the number of particular bacteria and HDL were found after $H$. pylori eradication (Prevotella copri: $r=0.34, p=0.037$; Prevotella stercorea: $r=-0.34, p=0.04$; Lachnobacterium $r=0.36$ $p=0.028$; Delsufovibrio $r=0.48, p=0.003$ ). Changes in the abundance of Bacteroides were associated with changes in HDL/LDL ratio after $H$. pylori eradication $(r=-0.364$, $p=0.029)$. In multivariate regression analysis, changes in Delsufovibrio $\left(R^{2}=0.130, \beta=0.39, p=0.017\right.$, and $R^{2}=0.082$, $\beta=0.44, p=0.015$, after including age, sex, and BMI) and Rikenellaceae $\left(R^{2}=0.098, \beta=-0.353, p=0.035\right.$, and $R^{2}=$ $0.022, \beta=-0.350, p=0.045$, after including age, sex, and BMI) predicted the proportion of changes in the HDL level, in patients after eradication treatment (Figure 2).

\section{DISCUSSION}

There are no previous studies that evaluate changes in microbiota related to lipid metabolism in otherwise healthy subjects prepost-H. pylori eradication. Alterations in blood cholesterol levels (high LDL and low HDL) are major risk factors for metabolic syndrome and cardiovascular disease (17). Antibiotic treatment for $H$. pylori eradication increased the HDL, reaching values similar to controls, in agreement with previous studies (18), 

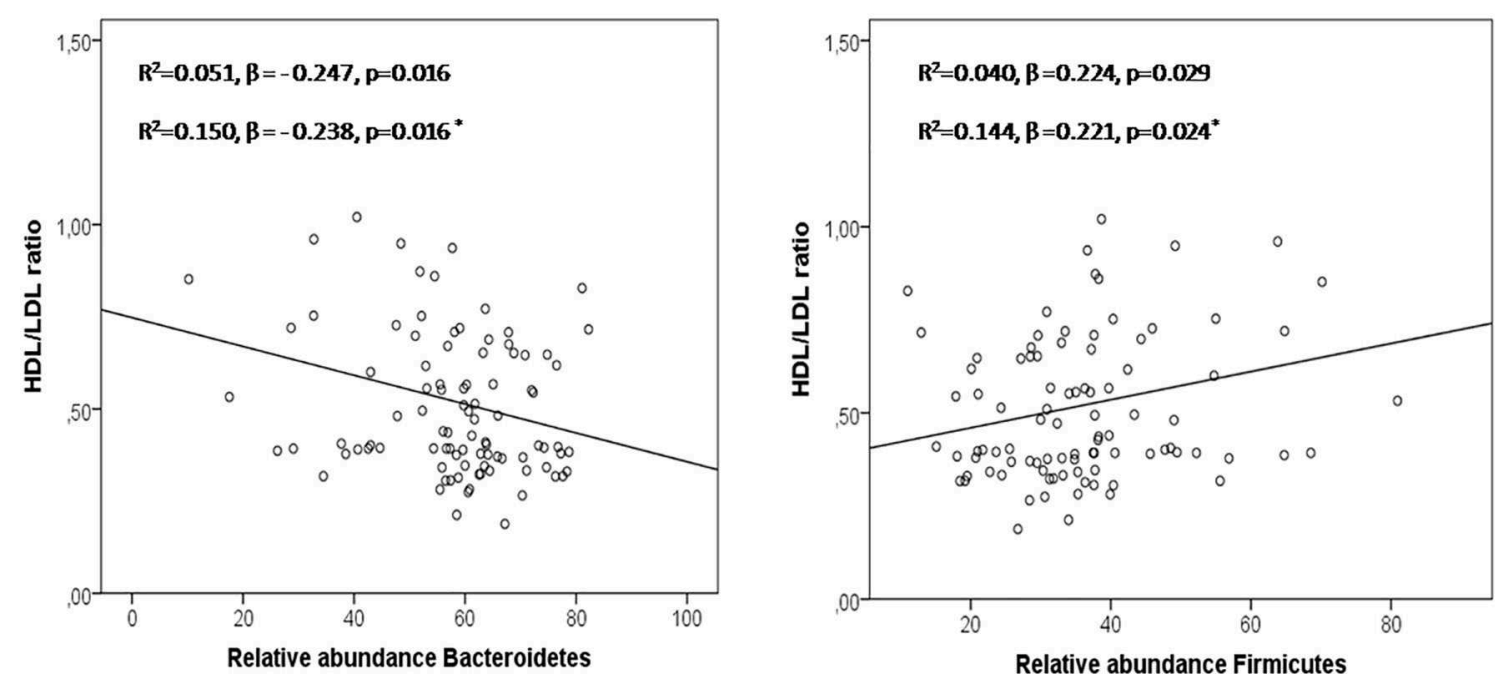

FIGURE 1 | Bacteroidetes and Firmicutes in the prediction of HDL/LDL ratio in a linear regression model including the 3 groups studied. Asterisk: regression model adjusted by age, sex, and BMI.

TABLE 2 | Association between intestinal bacteria and HDL/LDL ratio.

\begin{tabular}{lccccccc}
\hline Genus/Species & \multicolumn{3}{c}{ Model 1 } & & \multicolumn{3}{c}{ Model 2 } \\
\cline { 2 - 5 } \cline { 7 - 8 } & $\boldsymbol{R}^{\mathbf{2}}$ & $\boldsymbol{\beta}$ & $\boldsymbol{p}$ & $\boldsymbol{R}^{\mathbf{2}}$ & $\boldsymbol{\beta}$ & $\boldsymbol{P}$ \\
\hline B. coprophilus & 0.049 & -0.243 & 0.018 & 0.148 & -0.233 & 0.018 \\
Eubacterium & 0.043 & -0.231 & 0.025 & 0.148 & 0.235 & 0.018 \\
E. biforme & 0.045 & -0.236 & 0.021 & 0.149 & -0.237 & 0.017 \\
\hline
\end{tabular}

Dependent variable: HDL/LDL ratio. Model 1: linear univariate regression model. Model 2: linear multivariate regression model adjusted by age, sex, and BMI.

and both eradication and infection produced changes in the microbiota, data described in detail in our previous study (11). More interestingly, this is the first time that variations in blood lipid levels have been related to specific bacteria from the microbiome independent of age, sex, and BMI resulting $H$. pylori infection and eradication, suggesting that gut microbiota may affect specific aspects of lipid metabolism. Further, many of the identified taxa related to lipid metabolism are novel findings.

The use of broad spectrum antibiotics have been widely established to cause community-wide microbiota perturbations (19). In our study, H. pylori infection and its eradication with amoxicillin and clarithromycin for 10 days induced a dysbiosis affecting microbial diversity, something that has been associated to metabolic functions of intestinal bacteria. Low microbial functional richness has been associated to metabolic disease, including levels of fasting triglyceride, LDL and a raise in inflammation markers (20). In this sense, our study associated unfavorable lipid profiles (high LDL and low HDL level) with a low microbial richness in metabolically healthy subjects. In addition, this unfavorable lipid profile (lower HDL/LDL ratio) was associated with the phylum Bacteroidetes. As opposed, Firmicutes was associated with a favorable lipid profile (higher HDL/LDL ratio). The association between blood lipids and gut microbiome has been previously reported $(2,21)$. Firmicutes and Bacteroidetes are the two main bacteria phyla and they are implicated in the homeostasis of the host and fat accumulation. Interestingly, in our study, for the first time, Bacteroidetes and Firmicutes predicted HDL and LDL levels, regardless of age, sex, and BMI.

The raise in Bacteroidetes and the disminution in Firmicutes associated to the $H$. pylori infection and antibiotic-treatment could affect the production of key metabolites for the host, including SCFAs, bile acids, and lipopolysaccharide (LPS). In fact, acetate and propionate are produced mainly by members of Bacteroidetes while butyrate is typically produced by members of Firmicutes (22). Alterations in the microbiota as well as in their metabolites influence key processes as energy harvesting, the activation of the immune system, modulation of the chronic inflammation through the modification of the intestinal barrier permeability and perturbation of the reverse cholesterol transport, triggering in a higher susceptibility for some metabolic diseases (23).

The impact of the antibiotherapy, amoxicillin, and clarithromycin, on lipid metabolism as a result of the disturbance on the microbiota is an area of interest with limited knowledge at present. In this sense, we show a negative association between changes in the abundance of $P$. stercorea and HDL level and a positive association between changes in the abundance of P. copri, Lachonobacterium, Delsufovibrio, and HDL level at 2 months after completing antibiotic treatment.

Lachnospiraceae family has been specially associated with LDL (2) and development of metabolic disorders (24). Our study associated Lachnospiraceae/Lachonobacterium with HDL. Lachnospiraceae family is known by its capacity to metabolize complex polysaccharides to SCFAs, as butyrate, acetate, and propionate (25). Butyrate controls gene expression through epigenetic control. Buryrate is a key energy source for the 

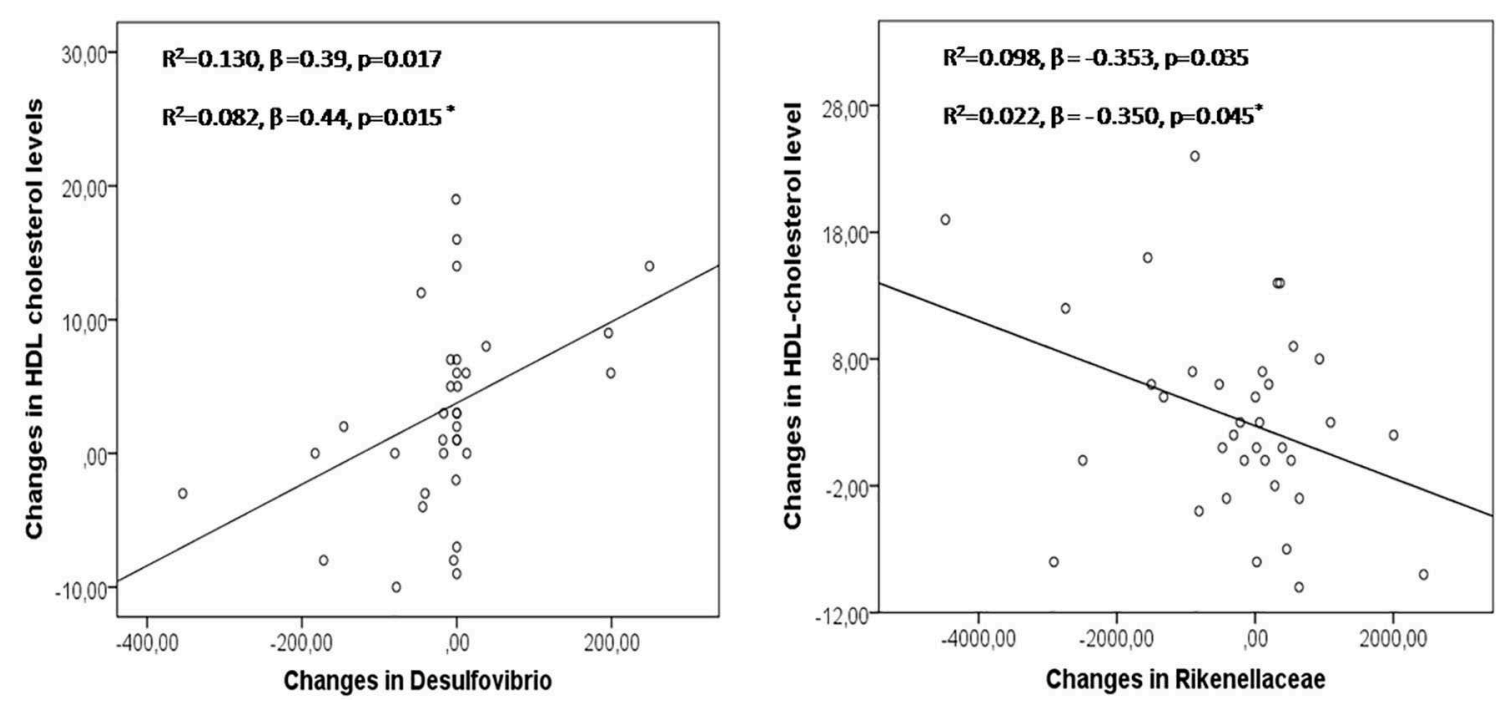

FIGURE 2 | Changes in Desulfovibrio and Rikenellaceae in the prediction of modifications in HDL levels after $H$ pylori eradication treatment. Asterisk: linear regression model adjusted by age, sex, and BMI.

colonic mucosa (26). Acetate is implicated in the de novo hepatic lipogenesis through acetyl-coA and fatty acid synthase (FAS), while propionate balances lipogenesis (27). Moreover, acetate is needed for the biosynthesis of cholesterol in liver. Thus, changes in the acetate/propionate ratio could play a crucial role in the regulation of lipid and cholesterol metabolism (28).

More interestingly is that alterations in Delsufovibrio, from the Proteobacteria phylum, and Rikenellaceae, from the Bacteroidetes phylum, predicted changes in HDL levels at 2 months after completing antibiotic treatment. To date, lower abundance of Desulfovibrio has been associated with obesity, blood pressure, insulin, and LDL (29). Desulfovibrio also has been inversely correlated to BMI (29). We showed, for the first time, that Delsufovibrio positively contributed to the HDL level independently of BMI, suggesting that this bacterium affects blood lipids partly. Bacteria such as Delsufovibrio and P. copri are producers of lipopolysaccharide (LPS). LPS is a ligand for tolllike receptor 4 (TLR4), which activates innate immune system, something that could trigger in a pro-inflammatory status (30). The inflammatory response to microbial stimulus may induce metabolic modifications. Low grade systemic inflammation associated with bacterial LPS-induced endotoxaemia as well as a gut permeability increase have been associated with elevated plasma HDL (31). This finding could be controversial, as obesity, metabolic syndrome and type- 2 diabetes are characterized by and impaired barrier function and local systemic inflammation (32), and they are characterized by a low HDL. On the other hand, products secreted by Desulfovibrio can up-regulate CD36 expression (33). CD36 is a critical regulator of lipid absorption, which has been positively correlated with HDL (34). All of these works would be in accordance with our data, and could help explain the results obtained in this study. In addition, we have reported an inverse relationship between Rikenellaceae, glucose (AUC) (11) and HDL levels at
2 months antibiotic treatment. Rikenellaceae is able to use the environmental glucose for acetate production (35). Acetic acid stimulates "de novo" lipogenesis and cholesterogenesis in the liver (36). The increase in the production of SCFAs as well as the elimination of glucose from the environment by this bacterium could explain part of the observed results. In addition, tudies have been showed a possible role of Clostridiales (including Lachonobacterium) and Bacteroidales (including Rikenellaceae, P. copri, and P. stercorea) in the metabolism of lipids, through the metabolic pathway of bile acids (37). In fact, secondary bile acids from the bacteria metabolism are absorbed being able to systemically modulate lipid and glucose metabolisms through nuclear or G protein-coupled receptors (GPCRs), such as FXR or TGR5 (37).

The current study, although it has some strengths, there are also a few limitations to be considered. The targeted sequencing of the16S rRNA, although it permits know who is who, has the impediment to identify specific species and strains as well as gives little information on bacterial functions. Moreover, although previous sample size calculations were performed to ensure a real approach, sample size could be augmented. The lack of a group of subjects without an $H$. pylori infection exposed to the eradication treatment could be indicated as another limitation, although it was not performed because of ethical reasons. This group could have provided more detailed data on the role of antibiotic treatment. Lastly, the follow-up period was based on a visit at 2 months the administration of the antibiotic treatment. The introduction of new time-points after 2 months could permit us know more about the dynamics suffered after eradication treatment.

In summary, antibiotic eradication treatment for $H$. pylori could increase HDL levels and affected the gut microbiota composition. The results obtained indicate that intestinal microbiome, specifically bacteria such as P. copri, 
Lachonobacterium and Delsufovibrio, Rikenellaceae could plays a role in lipid metabolism.

\section{DATA AVAILABILITY STATEMENT}

The datasets generated for this study can be found in the SRA database public repository from NCBI within the BioProject accession number PRJNA517270.

\section{ETHICS STATEMENT}

The studies involving human participants were reviewed and approved by Medical Ethics Committee at Virgen de la Victoria University Hospital and conducted in accordance with the Declaration of Helsinki. Written informed consent was provided by all participants, who also were verbally informed of the characteristics on the study. The patients/participants provided their written informed consent to participate in this study.

\section{AUTHOR CONTRIBUTIONS}

GM-N was person in charge of the metagenomic laboratory analysis, statistical analysis and interpretation of data, as well as the drafting, and reviewing of the manuscript. IC-P was person in charge of the recruitment of the patients and their follow-up and contributed to the design of the study and the revision of the manuscript. MR-R and JF-G also participated in the recruitment

\section{REFERENCES}

1. Wang Z, Koonen D, Hofker M, Fu J. Gut microbiome and lipid metabolism: from associations to mechanisms. Curr Opin Lipidol. (2016) 27:21624. doi: 10.1097/MOL.0000000000000308

2. Fu J, Bonder MJ, Cenit MC, Tigchelaar EF, Maatman A, Dekens JA, et al. The gut microbiome contributes to a substantial proportion of the variation in blood lipids. Circ Res. (2015) 117:817-24. doi: 10.1161/CIRCRESAHA.115. 306807

3. Martin FP, Dumas ME, Wang Y, Legido-Quigley C, Yap IK, Tang H, et al. A top-down systems biology view of microbiome-mammalian metabolic interactions in a mouse model. Mol Syst Biol. (2007) 3:112. doi: $10.1038 / \mathrm{msb} 4100153$

4. Arora T, Backhed F. The gut microbiota and metabolic disease: current understanding and future perspectives. J Intern Med. (2016) 280:33949. doi: 10.1111/joim.12508

5. Morrison DJ, Preston T. Formation of short chain fatty acids by the gut microbiota and their impact on human metabolism. Gut Microbes. (2016) 7:189-200. doi: 10.1080/19490976.2015.1134082

6. Alphonse PA, Jones PJ. Revisiting human cholesterol synthesis and absorption: the reciprocity paradigm and its key regulators. Lipids. (2016) 51:519-36. doi: 10.1007/s11745-015-4096-7

7. Jakobsson HE, Jernberg C, Andersson AF, Sjolund-Karlsson M, Jansson JK, Engstrand L. Short-term antibiotic treatment has differing long-term impacts on the human throat and gut microbiome. PLoS ONE. (2010) 5:e9836. doi: 10.1371/journal.pone.0009836

8. Glynn JR. Helicobacter pylori and the heart. Lancet. (1994) 344:146. doi: 10.1016/S0140-6736(94) 92754-5

9. Yap TW, Gan HM, Lee YP, Leow AH, Azmi AN, Francois F, et al. Helicobacter pylori eradication causes perturbation of the human gut microbiome in and follow-up of the patients, as well as reviewed the manuscript. MC-P performed laboratory analysis and participated in the revision of the manuscript. FC participated in the study design and reviewed the manuscript. IM-I assessed the bioinformatic analysis and critical revision of the manuscript. FT contributed to the study concept and design, interpretation of data, reviewed, and critically revised the article. All authors contributed to the article and approved the submitted version.

\section{FUNDING}

GM-N was supported by a Juan de la Cierva, Formación contract (FJCI-2017-34349) from the Spanish Ministry of Science (Spain). IC-P was now the recipient of a postdoctoral grant (Juan Rodés JR19/00054) from the Instituto de Salud Carlos III (ISCIII) and co-funded by Fondo Europeo de Desarrollo Regional-FEDER. MC-P was supported by a Juan de la Cierva, Formación contract (FJCI-2017-32194) from the Spanish Ministry of Science (Spain). FC was supported by a Nicolas Monardes contract (C-0032-2016) from Servicio Andaluz de Salud (SAS). JF-G was supported by a research contract from SAS (B-0003-2017). IM-I was supported by a MS type I contract (CP16/00163) from the ISCIII and cofunded by Fondo Europeo de Desarrollo Regional-FEDER. This work has been supported in part by a grant from the ISCIII co-funded by Fondo Europeo de Desarrollo Regional-FEDER, PI14/00082, PI15/01114, PI18/01160 Madrid, Spain, and by the Centros de Investigación Biomédica en Red (CIBER) of the Institute of Health Carlos III (ISCIII) (CB06/03/0018).

young adults. PLoS ONE. (2016) 11:e0151893. doi: 10.1371/journal.pone.01 51893

10. Cornejo-Pareja I, Martin-Nunez GM, Roca-Rodriguez MM, Cardona F, CoinAraguez L, Sanchez-Alcoholado L, et al. H. pylori eradication treatment alters gut microbiota and GLP-1 secretion in humans. J Clin Med. (2019) 8:451. doi: $10.3390 / \mathrm{jcm} 8040451$

11. Martin-Nunez GM, Cornejo-Pareja I, Coin-Araguez L, Roca-Rodriguez MDM, Munoz-Garach A, Clemente-Postigo M, et al. H. pylori eradication with antibiotic treatment causes changes in glucose homeostasis related to modifications in the gut microbiota. PLoS ONE. (2019) 14:e0213548. doi: 10.1371/journal.pone.0213548

12. Baumgartner S, Reijnders D, Konings M, Groen AK, Lutjohann D, Goossens $\mathrm{GH}$, et al. The effects of amoxicillin and vancomycin on parameters reflecting cholesterol metabolism. Chem Phys Lipids. (2017) 207:23945. doi: 10.1016/j.chemphyslip.2017.06.006

13. Moreno-Indias I, Sanchez-Alcoholado L, Garcia-Fuentes E, Cardona F, Queipo-Ortuno MI, Tinahones FJ. Insulin resistance is associated with specific gut microbiota in appendix samples from morbidly obese patients. Am J Transl Res. (2016) 8:5672-84.

14. Moreno-Indias I, Sanchez-Alcoholado L, Perez-Martinez P, Andres-Lacueva C, Cardona F, Tinahones F, et al. Red wine polyphenols modulate fecal microbiota and reduce markers of the metabolic syndrome in obese patients. Food Funct. (2016) 7:1775-87. doi: 10.1039/C5FO00886G

15. Lohman TG, Reynaldo AF, Martorell R. Standardization of Anthropometric Measurements. Champaign, IL: Human Kinetics Books (1988).

16. Klindworth A, Pruesse E, Schweer T, Peplies J, Quast C, Horn M, et al. Evaluation of general $16 \mathrm{~S}$ ribosomal RNA gene PCR primers for classical and next-generation sequencing-based diversity studies. Nucleic Acids Res. (2013) 41:e1. doi: 10.1093/nar/gks808

17. Cullen P, Assmann G. High risk strategies for atherosclerosis. Clin Chim Acta. (1999) 286:31-45. doi: 10.1016/S0009-8981(99)00092-3 
18. Scharnagl H, Kist M, Grawitz AB, Koenig W, Wieland H, Marz W. Effect of Helicobacter pylori eradication on high-density lipoprotein cholesterol. Am J Cardiol. (2004) 93:219-20. doi: 10.1016/j.amjcard.2003.09.045

19. Willing BP, Russell SL, Finlay BB. Shifting the balance: antibiotic effects on host-microbiota mutualism. Nat Rev Microbiol. (2011) 9:23343. doi: $10.1038 /$ nrmicro2536

20. Le Chatelier E, Nielsen T, Qin J, Prifti E, Hildebrand F, Falony G, et al. Richness of human gut microbiome correlates with metabolic markers. Nature. (2013) 500:541-6. doi: 10.1038/nature12506

21. Karlsson FH, Tremaroli V, Nookaew I, Bergstrom G, Behre CJ, Fagerberg B, et al. Gut metagenome in European women with normal, impaired and diabetic glucose control. Nature. (2013) 498:99-103. doi: 10.1038/nature12198

22. Chakraborti CK. New-found link between microbiota and obesity. World $J$ Gastrointest Pathophysiol. (2015) 6:110-9. doi: 10.4291/wjgp.v6.i4.110

23. Kasubuchi M, Hasegawa S, Hiramatsu T, Ichimura A, Kimura I. Dietary gut microbial metabolites, short-chain fatty acids, and host metabolic regulation. Nutrients. (2015) 7:2839-49. doi: 10.3390/nu7042839

24. Cho I, Yamanishi S, Cox L, Methe BA, Zavadil J, Li K, et al. Antibiotics in early life alter the murine colonic microbiome and adiposity. Nature. (2012) 488:621-6. doi: 10.1038/nature11400

25. Eren AM, Sogin ML, Morrison HG, Vineis JH, Fisher JC, Newton RJ, et al. A single genus in the gut microbiome reflects host preference and specificity. ISME J. (2015) 9:90-100. doi: 10.1038/ismej.2014.97

26. Meijer K, De Vos P, Priebe MG. Butyrate and other short-chain fatty acids as modulators of immunity: what relevance for health? Curr Opin Clin Nutr Metab Care. (2010) 13:715-21. doi: 10.1097/MCO.0b013e32833eebe5

27. Weitkunat K, Schumann S, Nickel D, Kappo KA, Petzke KJ, Kipp AP, et al. Importance of propionate for the repression of hepatic lipogenesis and improvement of insulin sensitivity in high-fat diet-induced obesity. Mol Nutr Food Res. (2016) 60:2611-21. doi: 10.1002/mnfr.201600305

28. Favier ML, Moundras C, Demigne C, Remesy C. Fermentable carbohydrates exert a more potent cholesterol-lowering effect than cholestyramine. Biochim Biophys Acta. (1995) 1258:115-21. doi: 10.1016/0005-2760(95)00107-N

29. De Moraes AC, Fernandes GR, Da Silva IT, Almeida-Pititto B, Gomes EP, Pereira AD, et al. Enterotype may drive the dietary-associated cardiometabolic risk factors. Front Cell Infect Microbiol. (2017) 7:47. doi: 10.3389/fcimb.2017.00047

30. Velloso LA, Folli F, Saad MJ. TLR4 at the crossroads of nutrients, gut microbiota, and metabolic inflammation. Endocr Rev. (2015) 36:24571. doi: 10.1210/er.2014-1100
31. Robertson MD, Pedersen C, Hinton PJ, Mendis A, Cani PD, Griffin BA. Elevated high density lipoprotein cholesterol and low grade systemic inflammation is associated with increased gut permeability in normoglycemic men. Nutr Metab Cardiovasc Dis. (2018) 28:1296-303. doi: 10.1016/j.numecd.2018.07.006

32. Bischoff SC, Barbara G, Buurman W, Ockhuizen T, Schulzke JD, Serino $\mathrm{M}$, et al. Intestinal permeability-a new target for disease prevention and therapy. BMC Gastroenterol. (2014) 14:189. doi: 10.1186/s12876-0140189-7

33. Petersen C, Bell R, Klag KA, Lee SH, Soto R, Ghazaryan A, et al. T cellmediated regulation of the microbiota protects against obesity. Science. (2019) 365:eaat9351. doi: 10.1126/science.aat9351

34. Rac ME, Safranow K, Garanty-Bogacka B, Dziedziejko V, Kurzawski G, Goschorska $\mathrm{M}$, et al. CD36 gene polymorphism and plasma sCD36 as the risk factor in higher cholesterolemia. Arch Pediatr. (2018) 25:17781. doi: 10.1016/j.arcped.2018.01.008

35. Su XL, Tian Q, Zhang J, Yuan XZ, Shi XS, Guo RB, et al. Acetobacteroides hydrogenigenes gen. nov, sp nov, an anaerobic hydrogen-producing bacterium in the family Rikenellaceae isolated from a reed swamp. Int J Syst Evol Microbiol. (2014) 64:2986-91. doi: 10.1099/ijs.0.063917-0

36. Wong JM, De Souza R, Kendall CW, Emam A, Jenkins DJ. Colonic health: fermentation and short chain fatty acids. J Clin Gastroenterol. (2006) 40:23543. doi: 10.1097/00004836-200603000-00015

37. Joyce SA, Macsharry J, Casey PG, Kinsella M, Murphy EF, Shanahan F, et al. Regulation of host weight gain and lipid metabolism by bacterial bile acid modification in the gut. Proc Natl Acad Sci USA. (2014) 111:74216. doi: $10.1073 /$ pnas. 1323599111

Conflict of Interest: The authors declare that the research was conducted in the absence of any commercial or financial relationships that could be construed as a potential conflict of interest.

Copyright (C) 2020 Martín-Núñez, Cornejo-Pareja, Roca-Rodríguez, ClementePostigo, Cardona, Fernández-García, Moreno-Indias and Tinahones. This is an open-access article distributed under the terms of the Creative Commons Attribution License (CC BY). The use, distribution or reproduction in other forums is permitted, provided the original author(s) and the copyright owner(s) are credited and that the original publication in this journal is cited, in accordance with accepted academic practice. No use, distribution or reproduction is permitted which does not comply with these terms. 